Advances in Algebra.

ISSN 0973-6964 Volume 6, Number 1 (2011), pp. 5-12

(C) Research India Publications

http://www.ripublication.com/aa.htm

\title{
On Generalized Fuzzy Graph Structures II
}

\author{
Dinesh T. and Ramakrishnan T.V. \\ Department of Mathematical Sciences, Kannur University, \\ Mangattuparamba, Kannur University Campus P.O.-670 567, \\ Kerala, India \\ E-mail:dineshthek@gmail.com
}

\begin{abstract}
In an earlier paper, we introduced the concept of a fuzzy graph structure analogous to the concept of graph structure given by E. Sampathkumar, in Bull. Kerala Math. Assoc., Vol 3, No.2 (2006 December), 65-123. In this paper, new concepts like $\rho_{i}$-bridge and $\rho_{i}$-cutvertex are introduced. Some results are obtained. We continue the study in two other papers which have been communicated.
\end{abstract}

AMS Subject Classification: 05C72, 05C40.

Keywords: $\rho_{i}$-bridge, $\rho_{i}$-cutvertex.

\section{Introduction}

The notion of fuzzy sets introduced by L.A. Zadeh in 1965, involves the concept of a membership function defined on a universal set. The value of the membership function lies in $[0,1]$. Using this concept, the idea of fuzzy graph was introduced by A. Rosenfeld in 1975 (cf. [4]).

A new concept, namely, graph structure has been introduced by E. Sampathkumar in [5] which, in particular, is a generalisation of the notions like graphs, signed graphs and edge - coloured graphs with colourings. According to him, $G=\left(V, R_{1}, R_{2}, \ldots, R_{k}\right)$ is a graph structure if $\mathrm{V}$ is a nonempty set and $R_{1}, R_{2}, \ldots, R_{k}$ are relations on $\mathrm{V}$ which are mutually disjoint such that each $R_{i}, i=1,2,3, \ldots, k$, is symmetric and irreflexive.

This is the motivation for the study of fuzzy graph structures. In an earlier paper [1], we introduced new concepts like $\rho_{i}$-edge, $\rho_{i}$-path, $\rho_{i}$-cycle, $\rho_{i}$-tree, $\rho_{i}$-forest, fuzzy $\rho_{i}$-cycle, fuzzy $\rho_{i}$-tree, fuzzy $\rho_{i}$-forest and $\rho_{i}$-connectedness. Here some other concepts like $\rho_{i}$-bridges and $\rho_{i}$-cut vertices are introduced and studied.

Essential preliminaries are given in section 2. For more details in Graph Theory, reference may be made to [2], for Fuzzy Graph Theory, to [3] and for Graph Structures, to [5]. 


\section{Preliminaries}

We recall some definitions on fuzzy graph structure as given in [1].

Definition 2.1. Let $G=\left(V, R_{1}, R_{2}, \ldots, R_{k}\right)$ be a graph structure and $\mu, \rho_{1}, \rho_{2}, \ldots, \rho_{k}$ be fuzzy subsets of $\left(V, R_{1}, R_{2}, \ldots, R_{k}\right)$ respectively such that

$$
\rho_{i}(x, y) \leq \mu(x) \wedge \mu(y) \forall x, y \in V
$$

and $i=1,2, \ldots, k$. Then $\tilde{G}=\left(\mu, \rho_{1}, \rho_{2}, \ldots, \rho_{k}\right)$ is a fuzzy graph structure of $\mathrm{G}$.

Convention: Throughout this paper, unless otherwise stated, $\mathrm{G}$ and $\tilde{G}$ will have the above meaning.

Example 2.2. Let

$$
\begin{aligned}
V & =\left\{x_{0}, x_{1}, x_{2}, x_{3}, x_{4}, x_{5}\right\}, \\
R_{1} & =\left\{\left(x_{0}, x_{1}\right),\left(x_{1}, x_{0}\right),\left(x_{0}, x_{2}\right),\left(x_{2}, x_{0}\right),\left(x_{3}, x_{4}\right),\left(x_{4}, x_{3}\right)\right\}, \\
R_{2} & =\left\{\left(x_{1}, x_{2}\right),\left(x_{2}, x_{1}\right),\left(x_{4}, x_{5}\right),\left(x_{5}, x_{4}\right)\right\}, R_{3}=\left\{\left(x_{2}, x_{3}\right),\left(x_{3}, x_{2}\right)\right\}
\end{aligned}
$$

and $G=\left(V, R_{1}, R_{2}, R_{3}\right)$ be a graph structure. Let $\tilde{G}=\left(\mu, \rho_{1}, \rho_{2}, \rho_{3}\right)$ where

$$
\begin{aligned}
\mu\left(x_{0}\right) & =0.8, \mu\left(x_{1}\right)=0.9, \mu\left(x_{2}\right)=0.6, \mu\left(x_{3}\right)=0.5, \mu\left(x_{4}\right)=0.6, \mu\left(x_{5}\right)=0.7 \\
\rho_{1}\left(x_{0}, x_{1}\right) & =0.8=\rho_{1}\left(x_{1}, x_{0}\right), \rho_{1}\left(x_{0}, x_{2}\right)=0.5=\rho_{1}\left(x_{2}, x_{0}\right), \\
\rho_{1}\left(x_{3}, x_{4}\right) & =0.4=\rho_{1}\left(x_{4}, x_{3}\right), \rho_{2}\left(x_{1}, x_{2}\right)=0.6=\rho_{2}\left(x_{2}, x_{1}\right), \\
\rho_{2}\left(x_{4}, x_{5}\right) & =0.5=\rho_{2}\left(x_{5}, x_{4}\right), \rho_{3}\left(x_{2}, x_{3}\right)=0.3=\rho_{3}\left(x_{3}, x_{2}\right), \\
\rho_{3}\left(x_{0}, x_{5}\right) & =0.5=\rho_{3}\left(x_{5}, x_{0}\right)
\end{aligned}
$$

Here notice that $\rho_{1}\left(x_{0}, x_{1}\right) \leq \wedge\left(\mu\left(x_{0}\right), \mu\left(x_{1}\right)\right)$ and so on. Therefore, $\tilde{G}$ is a fuzzy graph structure of $G$. Now we recall some basic notions of fuzzy graph structures. In all these, $i \in\{1,2, \ldots, k\}$.

Definition 2.3. $\tilde{F}=\left(\mu, \tau_{1}, \tau_{2}, \ldots, \tau_{k}\right)$ is a partial fuzzy spanning subgraph structure of $\tilde{G}=\left(\mu, \rho_{1}, \rho_{2}, \ldots, \rho_{k}\right)$ if $\tau_{r} \subseteq \rho_{r}$ for $r=1,2, \ldots, k$.

Definition 2.4. Let $\mathrm{G}$ be a graph structure and $\tilde{G}$ be a fuzzy graph structure of $\mathrm{G}$. If $(x, y) \in \operatorname{supp}\left(\rho_{i}\right)$, then $(\mathrm{x}, \mathrm{y})$ is said to be a $\rho_{i}-e d g e$ of $\tilde{G}$.

In example $2.2,\left(x_{0}, x_{1}\right),\left(x_{0}, x_{2}\right),\left(x_{3}, x_{4}\right)$ are $\rho_{1}$-edges, $\left(x_{1}, x_{2}\right),\left(x_{4}, x_{5}\right)$ are $\rho_{2}-$ edges and $\left(x_{2}, x_{3}\right),\left(x_{0}, x_{5}\right)$ are $\rho_{3}$-edges.

Definition 2.5. A $\rho_{i}$-path of a fuzzy graph structure $\tilde{G}$ is a sequence of vertices, $x_{0}$, $x_{1}, \ldots, x_{n}$ which are distinct (except possibly $\left.x_{n}, x_{0}\right)$ such that $\left(x_{j-1}, x_{j}\right)$ is a $\rho_{i}$-edge for all $j \in\{1,2, \ldots, n\}$.

In example 2.2, $x_{1}, x_{0}, x_{2}$ is a $\rho_{1}$-path. 
Definition 2.6. Two vertices of a fuzzy graph structure $\tilde{G}$, joined by a $\rho_{i}$-path are said to be $\rho_{i}$-connected.

In example 2.2, $x_{1}$ and $x_{2}$ are $\rho_{1}$-connected and $x_{0}$ and $x_{5}$ are $\rho_{3}$-connected.

Definition 2.7. The strength of a $\rho_{i}$-path $x_{0}, x_{1}, \ldots, x_{n}$ of a fuzzy graph structure $\tilde{G}$ is

$\bigwedge_{j=1}^{n} \rho_{i}\left(x_{j-1}, x_{j}\right)$ for $i=1,2, \ldots, k$.

In example 2.2, strength of the $\rho_{1}$ - path $x_{1}, x_{0}, x_{2}$ is 0.5 .

Definition 2.8. In any fuzzy graph structure $\tilde{G}$,

$$
\rho_{i}^{2}(x, y)=\rho_{i} \circ \rho_{i}(x, y)=\bigvee_{z}\left\{\rho_{i}(x, z) \wedge \rho_{i}(z, y)\right\}
$$

and $\rho_{i}^{j}(x, y)=\rho_{i}^{j-1} \circ \rho_{i}(x, y), j=2,3, \ldots, m$ for any $\mathrm{m} \geq 2$. Also

$$
\rho_{i}^{\infty}(x, y)=\vee\left\{\rho_{i}^{j}(x, y): j=1,2, \ldots\right\} .
$$

Definition 2.9. $\tilde{G}$ is a $\rho_{i}$-cycle iff $\left(\operatorname{supp}(\mu), \operatorname{supp}\left(\rho_{1}\right), \operatorname{supp}\left(\rho_{2}\right), \ldots, \operatorname{supp}\left(\rho_{k}\right)\right)$ is an $R_{i}$ cycle (where an $R_{i}$-cycle is a sequence of vertices $x_{0}, x_{1}, \ldots, x_{n-1}, x_{n}=x_{0}$ in $V$ such that each $\left(x_{j-1}, x_{j}\right)$ is an $R_{i}$-edge for $j=1,2, \ldots, n$.

Example 2.10. Let $V=\left\{x_{0}, x_{1}, x_{2}, x_{3}, x_{4}, x_{5}\right\}, R_{1}=\left\{\left(x_{0}, x_{1}\right),\left(x_{1}, x_{0}\right),\left(x_{1}, x_{2}\right)\right.$, $\left.\left.\left.\left(x_{2}, x_{1}\right),\left(x_{2}, x_{3}\right),\left(x_{3}, x_{2}\right),\left(x_{0}, x_{3}\right)\right),\left(x_{3}, x_{0}\right)\right)\right\}, R_{2}=\left\{\left(x_{0}, x_{4}\right),\left(x_{4}, x_{0}\right)\right\}$, $R_{3}=\left\{\left(x_{3}, x_{4}\right),\left(x_{4}, x_{3}\right),\left(x_{4}, x_{5}\right),\left(x_{5}, x_{4}\right),\left(x_{5}, x_{0}\right),\left(x_{0}, x_{5}\right),\left(x_{6}, x_{7}\right),\left(x_{7}, x_{6}\right)\right\}$ and $G=\left(V, R_{1}, R_{2}, R_{3}\right)$ be a graph structure. Let $\tilde{G}=\left(\mu, \rho_{1}, \rho_{2}, \rho_{3}\right)$ where

$$
\begin{aligned}
\mu\left(x_{0}\right) & =0.8, \mu\left(x_{1}\right)=0.9, \mu\left(x_{2}\right)=0.6, \mu\left(x_{3}\right)=0.5, \mu\left(x_{4}\right)=0.6, \\
\mu\left(x_{5}\right) & =0.7, \mu\left(x_{6}\right)=0.6, \mu\left(x_{7}\right)=0.5 \\
\rho_{1}\left(x_{0}, x_{1}\right) & =0.8=\rho_{1}\left(x_{1}, x_{0}\right), \rho_{1}\left(x_{1}, x_{2}\right)=0.5=\rho_{1}\left(x_{2}, x_{1}\right), \\
\rho_{1}\left(x_{2}, x_{3}\right) & =0.5=\rho_{1}\left(x_{3}, x_{2}\right), \rho_{1}\left(x_{0}, x_{3}\right)=0.5=\rho_{1}\left(x_{3}, x_{0}\right), \\
\rho_{2}\left(x_{0}, x_{4}\right) & =0.6=\rho_{2}\left(x_{4}, x_{0}\right), \rho_{3}\left(x_{3}, x_{4}\right)=0.5=\rho_{3}\left(x_{4}, x_{3}\right), \\
\rho_{3}\left(x_{4}, x_{5}\right) & =0.6=\rho_{3}\left(x_{5}, x_{4}\right), \rho_{3}\left(x_{5}, x_{0}\right)=0.4=\rho_{3}\left(x_{0}, x_{5}\right), \\
\rho_{3}\left(x_{6}, x_{7}\right) & =0.5=\rho_{3}\left(x_{7}, x_{6}\right)
\end{aligned}
$$

In example $2.10,\left(x_{0}, x_{1}\right),\left(x_{1}, x_{2}\right),\left(x_{2}, x_{3}\right),\left(x_{0}, x_{3}\right)$ is a $\rho_{1}$-cycle.

Definition 2.11. $\tilde{G}$ is a fuzzy $\rho_{i}$-cycle iff $\left(\operatorname{supp}(\mu), \operatorname{supp}\left(\rho_{1}\right), \operatorname{supp}\left(\rho_{2}\right), \ldots, \operatorname{supp}\left(\rho_{k}\right)\right)$ is an $R_{i}$-cycle and there exists no unique $(\mathrm{x}, \mathrm{y})$ in $\operatorname{supp}\left(\rho_{i}\right)$ such that $\rho_{i}(x, y)=\wedge\left\{\rho_{i}(u, v) \mid\right.$ $\left.(u, v) \in \operatorname{supp}\left(\rho_{i}\right)\right\}$. 
In example $2.10,\left(x_{0}, x_{1}\right),\left(x_{1}, x_{2}\right),\left(x_{2}, x_{3}\right),\left(x_{0}, x_{3}\right)$ is a fuzzy $\rho_{1}$-cycle.

Definition 2.12. $\tilde{G}$ is a $\rho_{i}$-forest for $i=1,2, \ldots, k$ if its $\rho_{i}$-edges form an $R_{i}$-forest (where an $R_{i}$-forest is a Graph Structure which does not contain $R_{i}$-cycles).

In example $2.10,\left(x_{3}, x_{4}\right),\left(x_{4}, x_{5}\right),\left(x_{5}, x_{0}\right),\left(x_{6}, x_{7}\right)$ is a $\rho_{3}$-forest.

Definition 2.13. $\tilde{G}$ is a $\rho_{i}$-tree if it is a $\rho_{i}$-connected $\rho_{i}$-forest.

In example $2.10,\left(x_{3}, x_{4}\right),\left(x_{4}, x_{5}\right),\left(x_{5}, x_{0}\right)$ is a $\rho_{3}$-tree.

Definition 2.14. $\tilde{G}$ is a fuzzy $\rho_{i}$-forest if it has a partial fuzzy spanning subgraph structure $\tilde{F}_{i}=\left(\mu, \tau_{1}, \tau_{2}, \ldots, \tau_{k}\right)$ which is a $\tau_{i}$-forest where for all $\rho_{i}$-edges not in $\tilde{F}_{i}, \rho_{i}(x, y)<$ $\tau_{i}^{\infty}(x, y)$.

Definition 2.15. $\tilde{G}$ is a fuzzy $\rho_{i}$-tree for $i=1,2, \ldots, k$ if it has a partial fuzzy spanning subgraph structure $\tilde{F}_{i}=\left(\mu, \tau_{1}, \tau_{2}, \ldots, \tau_{k}\right)$ which is a $\tau_{i}$-tree where for all $\rho_{i}$-edges not in $\tilde{F}_{i}, \rho_{i}(x, y)<\tau_{i}^{\infty}(x, y)$.

Example 2.16. Let

$$
\begin{aligned}
V= & \left\{x_{0}, x_{1}, x_{2}, x_{3}, x_{4}, x_{5}\right\}, \\
R_{1}= & \left\{\left(x_{0}, x_{1}\right),\left(x_{1}, x_{0}\right),\left(x_{1}, x_{2}\right),\left(x_{2}, x_{1}\right),\left(x_{2}, x_{3}\right),\left(x_{3}, x_{2}\right),\left(x_{3}, x_{0}\right),\left(x_{0}, x_{3}\right),\right. \\
& \left.\left(x_{4}, x_{5}\right),\left(x_{5}, x_{4}\right)\right\}, R_{2}=\left\{\left(x_{0}, x_{4}\right),\left(x_{4}, x_{0}\right)\right\}
\end{aligned}
$$

and $G=\left(V, R_{1}, R_{2}\right)$ be a graph structure.

Let $\tilde{G}=\left(\mu, \rho_{1}, \rho_{2}\right)$ where

$$
\begin{aligned}
\mu\left(x_{0}\right) & =0.5, \mu\left(x_{1}\right)=0.6, \\
\mu\left(x_{2}\right) & =0.7, \mu\left(x_{3}\right)=0.8, \mu\left(x_{4}\right)=0.9, \mu\left(x_{5}\right)=0.8 \\
\rho_{1}\left(x_{0}, x_{1}\right) & =0.4=\rho_{1}\left(x_{1}, x_{0}\right), \rho_{1}\left(x_{1}, x_{2}\right)=0.5=\rho_{1}\left(x_{2}, x_{1}\right), \\
\rho_{1}\left(x_{2}, x_{3}\right) & =0.6=\rho_{1}\left(x_{3}, x_{2}\right), \rho_{1}\left(x_{0}, x_{3}\right)=0.3=\rho_{1}\left(x_{3}, x_{0}\right), \\
\rho_{1}\left(x_{4}, x_{5}\right) & =0.8=\rho_{1}\left(x_{5}, x_{4}\right), \rho_{2}\left(x_{0}, x_{4}\right)=0.5=\rho_{2}\left(x_{4}, x_{0}\right)
\end{aligned}
$$

$\tilde{G}$ is a fuzzy graph structure of $G$. Let $\tilde{F}_{1}=\left(\mu, \tau_{1}, \tau_{2}\right)$ be a partial fuzzy spanning subgraph structure of $\tilde{G}$ defined by

$$
\begin{aligned}
& \tau_{1}\left(x_{0}, x_{1}\right)=0.4=\tau_{1}\left(x_{1}, x_{0}\right), \\
& \tau_{1}\left(x_{1}, x_{2}\right)=0.5=\tau_{1}\left(x_{2}, x_{1}\right), \tau_{1}\left(x_{2}, x_{3}\right)=0.6=\tau_{1}\left(x_{3}, x_{2}\right), \\
& \tau_{1}\left(x_{4}, x_{5}\right)=0.8=\tau_{1}\left(x_{5}, x_{4}\right), \tau_{2}\left(x_{0}, x_{4}\right)=0.5=\tau_{2}\left(x_{4}, x_{0}\right)
\end{aligned}
$$

and $\tau_{1}=0, \tau_{2}=0$ for all other $\rho_{1}$ and $\rho_{2}$ edges. Then $\tilde{F}_{1}=\left(\mu, \tau_{1}, \tau_{2}\right)$ is a $\tau_{1}$-forest with $\rho_{1}(x, y)<\tau_{1}^{\infty}(x, y)$ for all $\rho_{1}$-edges not in $\tilde{F}_{1}$. Hence $\tilde{G}$ is a fuzzy $\rho_{1}$-forest.

In the above example, $\left(x_{0}, x_{1}\right),\left(x_{1}, x_{2}\right),\left(x_{2}, x_{3}\right),\left(x_{3}, x_{0}\right)$ form a fuzzy $\rho_{1}$-tree. 


\section{3. $\rho_{i}$-bridges and $\rho_{i}$-cut vertices}

We introduce some more new concepts like $\rho_{i}$-bridges and $\rho_{i}$-cut vertices of a fuzzy graph structure.

Definition 3.1. Let $(\mathrm{x}, \mathrm{y})$ be a $\rho_{i}$-edge of $\tilde{G}$. Let $\left(\mu, \rho_{1}^{\prime}, \rho_{2}^{\prime}, \ldots, \rho_{i}^{\prime}, \rho_{i+1}^{\prime}, \ldots, \rho_{k}^{\prime}\right)$ be a partial fuzzy spanning subgraph structure obtained by deleting $(\mathrm{x}, \mathrm{y})$ with $\rho_{i}^{\prime}(x, y)=0$ and $\rho_{i}^{\prime}\left(x_{1}, y_{1}\right)=\rho_{i}\left(x_{1}, y_{1}\right) \forall \rho_{i}$-edge $\left(x_{1}, y_{1}\right)$ other than $(\mathrm{x}, \mathrm{y})$. If $\rho_{i}^{\prime \infty}(u, v)<\rho_{i}^{\infty}(u, v)$ for some $(u, v) \in \operatorname{supp}\left(\rho_{i}\right)$, then $(\mathrm{u}, \mathrm{v})$ is a $\rho_{i}$-bridge.

Now we move on to some results using the concept of $\rho_{i}$-bridges.

Theorem 3.2. Let $\tilde{G}$ be a fuzzy graph structure. If $(\mathrm{x}, \mathrm{y})$ is a $\rho_{i}$-bridge, then $\rho_{i}^{\prime \infty}(x, y)<$ $\rho_{i}(x, y)$ where $\left(\mu, \rho_{1}^{\prime}, \rho_{2}^{\prime}, \ldots, \rho_{k}^{\prime}\right)$ is a partial fuzzy spanning subgraph structure obtained by deleting $(\mathrm{x}, \mathrm{y})$, for $i=1,2, \ldots, k$.

Proof. If possible, let $\rho_{i}^{\prime \infty}(x, y) \geq \rho_{i}(x, y)$ for some $\rho_{i}$-bridge $(\mathrm{x}, \mathrm{y})$. ie., there is a $\rho_{i}$-path of strength greater than $\rho_{i}(x, y)$ from $\mathrm{x}$ to $\mathrm{y}$ which does not involve $(\mathrm{x}, \mathrm{y})$. Thus any $\rho_{i}$-path involving $(\mathrm{x}, \mathrm{y})$ can be replaced by a $\rho_{i}$-path not involving $(\mathrm{x}, \mathrm{y})$ without reducing strength.

This is a contradiction to the fact that (x,y) is a $\rho_{i}$-bridge. Hence $\rho_{i}^{\infty}(x, y)<\rho_{i}(x, y)$ for $i=1,2, \ldots, k$.

Remark 3.3. Converse of the above result also holds. ie., if $\rho_{i}^{\prime \infty}(x, y)<\rho_{i}(x, y)$, then $(x, y)$ is a $\rho_{i}$-bridge.

Proof. If possible, let $(x, y)$ be not a $\rho_{i}$-bridge. Then $\rho_{i}^{\prime \infty}(x, y)=\rho_{i}^{\infty}(x, y) \geq \rho_{i}(x, y)$ which is a contradiction to our assumption.

Therefore $(x, y)$ is a $\rho_{i}$-bridge.

Theorem 3.4. Let $\tilde{G}$ be a fuzzy graph structure which is a fuzzy $\rho_{i}$-forest. Then the $\rho_{i}$-edges of the partial fuzzy spanning subgraph structure $\tilde{F}_{i}=\left(\mu, \tau_{1}, \tau_{2}, \ldots, \tau_{k}\right)$ which is a $\tau_{i}$-forest, are the $\rho_{i}$-bridges of $\tilde{G}$.

Proof. Case 1: $(x, y)$ is a $\rho_{i}$-edge not in $\tilde{F}_{i}$

By definition of a fuzzy $\rho_{i}$-forest, $\rho_{i}(x, y)<\tau_{i}^{\infty}(x, y) \leq \rho_{i}^{\prime \infty}(x, y)$

Therefore $(\mathrm{x}, \mathrm{y})$ is not a $\rho_{i}$-bridge by theorem 3.2.

Case 2: $(x, y)$ is a $\tau_{i}$-edge of $\tilde{F}_{i}$

If possible, let $(\mathrm{x}, \mathrm{y})$ be not a $\rho_{i}$-bridge. Then there exists a $\rho_{i}$-path $P_{i}$ from x to $\mathrm{y}$ not involving $(\mathrm{x}, \mathrm{y})$ with strength greater than or equal to $\rho_{i}(x, y) . P_{i}$ and $\tilde{F}_{i}$ form a $\rho_{i}$-cycle.

But $\tilde{F}_{i}$ does not contain $\tau_{i}$-cycles. Therefore, $P_{i}$ contains $\rho_{i}$-edges not in $\tilde{F}_{i}$.

Let $\left(u_{r}, v_{r}\right)$ be such a $\rho_{i}$-edge of $P_{i}$.

This can be replaced by a $\tau_{i}$-path $P_{i r}$ in $\tilde{F}_{i}$ having strength greater than $\rho_{i}\left(u_{r}, v_{r}\right)$ by definition of a fuzzy $\rho_{i}$-forest. 
Also $\rho_{i}\left(u_{r}, v_{r}\right) \geq \rho_{i}(x, y)$. All $\tau_{i}$-edges of $P_{i r}$ are stronger than $\rho_{i}\left(u_{r}, v_{r}\right)$ which is greater than or equal to $\rho_{i}(x, y)$.

Therefore $P_{i r}$ does not contain $(x, y)$. If it contains $(x, y)$, its strength will be less than or equal to $\tau_{i}(x, y) \leq \rho_{i}(x, y)$.

Thus we have a $\tau_{i}$-path in $\tilde{F}_{i}$ from $\mathrm{x}$ to y not involving $(x, y)$. This gives a $\tau_{i}$-cycle in $\tilde{F}_{i}$ and hence a $\rho_{i}$-cycle which is not possible. Hence $(\mathrm{x}, \mathrm{y})$ is a $\rho_{i}$-bridge. Thus the $\rho_{i}$-edges of $\tilde{F}_{i}$ are the $\rho_{i}$-bridges of $\tilde{G}$.

Now, we define a $\rho_{i}$-cut vertex. For that first we define the partial subgraph strucure $\left(\mu^{\prime}, \rho_{1}^{\prime}, \rho_{2}^{\prime}, \ldots, \rho_{k}^{\prime}\right)$.

Definition 3.5. $\tilde{G}^{\prime}=\left(\mu^{\prime}, \rho_{1}^{\prime}, \rho_{2}^{\prime}, \ldots, \rho_{k}^{\prime}\right)$ is the partial fuzzy subgraph structure obtained by the deletion of a vertex $w$ of $\tilde{G}$. ie., $\mu^{\prime}(w)=0$ and $\mu^{\prime}(u)=\mu(u) \forall u \neq w$ $\rho_{i}^{\prime}(w, v)=0 \forall v \in V$ and $\rho_{i}^{\prime}(u, v)=\rho_{i}(u, v) \forall(u, v) \neq(w, v), i=1,2, \ldots, k$.

Definition 3.6. A vertex $w$ of $\tilde{G}$ is a $\rho_{i}$-cut vertex if $\rho_{i}^{\prime \infty}(u, v)<\rho_{i}^{\infty}(u, v)$ for some $u, v$ with $u \neq w \neq v$ where $\mu^{\prime}$ and $\rho_{i}^{\prime}$ are as in definition 3.5.

Now we discuss some results on $\rho_{i}$-bridges and $\rho_{i}$-cut vertices.

Theorem 3.7. Let $\tilde{G}$ be a fuzzy graph structure with

$$
\tilde{G}^{*}=\left(\operatorname{supp}(\mu), \operatorname{supp}\left(\rho_{1}\right), \operatorname{supp}\left(\rho_{2}\right), \ldots, \operatorname{supp}\left(\rho_{k}\right)\right)
$$

a fuzzy $\rho_{i}$-cycle. If a vertex of $\tilde{G}$ is a $\rho_{i}$-cut vertex of $\tilde{G}$, then it is a common vertex of two $\rho_{i}$-bridges.

Proof. Consider a $\rho_{i}$-cut vertex $w$ of $\tilde{G}$. By the definition of a $\rho_{i}$-cut vertex, there exists two vertices $u$ and $v$ different from $w$ such that $w$ is on every strongest $u-v \rho_{i}$-path.

Given that $\tilde{G}^{*}$ is a fuzzy $\rho_{i}$-cycle. Then there exists only one strongest $\rho_{i}$-path $P_{i}$ from $u$ to $v$ containing $w$. In $P_{i}$, all $\rho_{i}$-edges are $\rho_{i}$-bridges.

Thus $w$ is common to two $\rho_{i}$-bridges.

Remark 3.8. Converse of the above result also holds as is evident from the next theorem.

Theorem 3.9. Let $\tilde{G}$ be a fuzzy graph structure. If $w$ is common to at least two $\rho_{i}$-bridges of $\tilde{G}$, then $w$ is a $\rho_{i}$-cut vertex.

Proof. Let $\left(u_{1}, w\right)$ and $\left(w, v_{2}\right)$ be two $\rho_{i}$-bridges with $w$ as the common vertex. Since $\left(u_{1}, w\right)$ is a $\rho_{i}$-bridge, it is on every strongest $u-v \rho_{i}$-path for some $u$ and $v$.

Case 1: $w \neq u, w \neq v$

In this case, $w$ is on every strongest $u-v \rho_{i}$-path for some $u$ and $v$. Then $w$ is a $\rho_{i}$-cut vertex.

Case 2: Either $w=u$ or $w=v$

In this case either $\left(u_{1}, w\right)$ is on every strongest $u-w \rho_{i}$-path or $\left(w, v_{2}\right)$ is on every strongest $w-v \rho_{i}$-path. If possible, let $w$ be not a $\rho_{i}$-cut vertex. 
By definition of $\rho_{i}$-cut vertex, there exists a strongest $\rho_{i}$-path not containing $w$ between any pair of vertices. Consider such a path $P_{i}$ joining $u_{1}$ and $v_{2}$. Then $P_{i},\left(u_{1}, w\right)$, $\left(w, v_{2}\right)$ form a $\rho_{i}$-cycle.

a) Let $u_{1}, w, v_{2}$ be not a strongest $\rho_{i}$-path.

Then $\left(u_{1}, w\right)$ or $\left(w, v_{2}\right)$ or both become the weakest $\rho_{i}$-edges of the above $\rho_{i}$-cycle consisting of $P_{i},\left(u_{1}, w\right)$ and $\left(w, v_{2}\right)$ since every $\rho_{i}$-edge of $P_{i}$ will be stronger than $\left(u_{1}, w\right)$ and $\left(w, v_{2}\right)$.

This is not possible since $\left(u_{1}, w\right)$ and $\left(w, v_{2}\right)$ are $\rho_{i}$-bridges.

b) Let $u_{1}, w, v_{2}$ also be a strongest $\rho_{i}$-path joining $u_{1}$ and $v_{2}$.

Then $\rho_{i}^{\infty}\left(u_{1}, v_{2}\right)=\rho_{i}\left(u_{1}, w\right) \wedge \rho_{i}\left(w, v_{2}\right)$ i.e., either $\left(u_{1}, w\right)$ or $\left(w, v_{2}\right)$ or both are the weakest $\rho_{i}$-edges of the above $\rho_{i}$-cycle because $P_{i}$ is as strong as $u_{1}, w, v_{2}$.

This is not possible because $u_{1}, w, v_{2}$ is a strongest $\rho_{i}$-path. Therefore, $w$ is a $\rho_{i}$-cut vertex.

Now we prove that the internal vertices of a $\rho_{i}$-tree of a fuzzy $\rho_{i}$-tree are the $\rho_{i}$-cut vertices.

Theorem 3.10. Let $\tilde{G}$ be a fuzzy $\rho_{i}$-tree for which $\tilde{F}_{i}=\left(\mu, \tau_{1}, \tau_{2}, \ldots, \tau_{k}\right)$ is a partial fuzzy spanning subgraph structure which is a $\tau_{i}$-tree and $\rho_{i}(x, y)<\tau_{i}^{\infty}(x, y) \forall(x, y)$ not in $\tilde{F}_{i}$. Then the internal vertices of $\tilde{F}_{i}$ are precisely the $\rho_{i}$-cut vertices of $\tilde{G}$.

Proof. Consider a vertex $w$ of $\tilde{F}_{i}$.

Case 1:w is not an end vertex of $\tilde{F}_{i}$

$w$ is common to two $\tau_{i}$-edges of $\tilde{F}_{i}$ at least and by theorem 3.4 , they are $\rho_{i}$-bridges of $\tilde{G}$. Then by theorem $3.9, w$ is a $\rho_{i}$-cut vertex.

Case 2: $w$ is an end vertex of $\tilde{F}_{i}$

If $w$ is a $\rho_{i}$-cut vertex, it lies on every strongest $\rho_{i}$-path and hence $\tau_{i}$-path joining $u$ and $v$ for some $u$ and $v$ in V. One of such $\tau_{i}$-paths lies in $\tilde{F}_{i}$. But $w$ is an end vertex of $\tilde{F}_{i}$. So this is not possible. So $w$ is not a $\rho_{i}$-cut vertex. i.e., the internal vertices of $\tilde{F}_{i}$ are precisely the $\rho_{i}$-cut vertices of $\tilde{G}$.

The above theorem leads us to the following corollary.

Corollary 3.11. A $\rho_{i}$-cut vertex, of a fuzzy graph structure $\tilde{G}$ which is a fuzzy $\rho_{i}$-tree, is common to at least two $\rho_{i}$-bridges.

\section{References}

[1] Dinesh, T. \& Ramakrishnan, T.V., On Generalised Fuzzy Graph Structures (Communicated).

[2] Harary, F., Graph Theory, Narosa Pub. House, 1995.

[3] Mordeson, J.N. \& Nair, P.S., Fuzzy Graphs and Fuzzy Hypergraphs, Physica-verlag, 2000. 
[4] Rosenfeld, A., Fuzzy Graphs in Fuzzy Sets and their Applications to Cognitive and Decision Processes (eds. L.A. Zadeh, K.S. Fu \& M. Shimura), pp 77-95, Acad. Press, New York, 1975.

[5] Sampathkumar, E., Generalized Graph Structures, Bull. Kerala Math. Assoc., Vol 3, No.2 (Dec 2006), 65-123. 